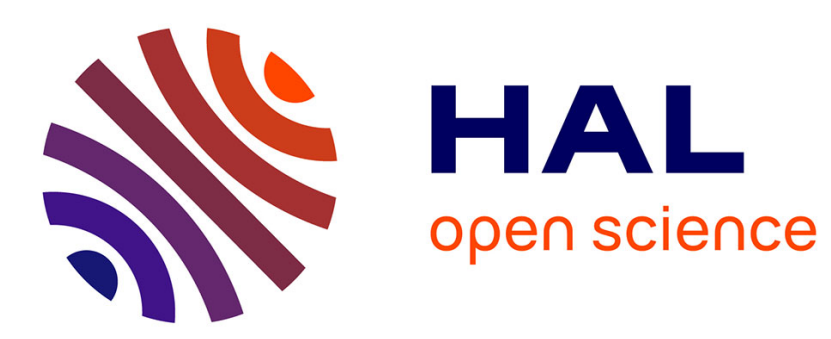

\title{
A Data-Driven approach for predicting the remaining useful life of steam generators
}

Hoang-Phuong Nguyen, William Fauriat, Enrico Zio, Jie Liu

\section{To cite this version:}

Hoang-Phuong Nguyen, William Fauriat, Enrico Zio, Jie Liu. A Data-Driven approach for predicting the remaining useful life of steam generators. 2018 3rd International Conference on System Reliability and Safety (ICSRS), Nov 2018, Barcelona, Spain. 10.1109/ICSRS.2018.00049 . hal-02194938

\section{HAL Id: hal-02194938 \\ https://hal-centralesupelec.archives-ouvertes.fr/hal-02194938}

Submitted on 26 Feb 2020

HAL is a multi-disciplinary open access archive for the deposit and dissemination of scientific research documents, whether they are published or not. The documents may come from teaching and research institutions in France or abroad, or from public or private research centers.
L'archive ouverte pluridisciplinaire HAL, est destinée au dépôt et à la diffusion de documents scientifiques de niveau recherche, publiés ou non, émanant des établissements d'enseignement et de recherche français ou étrangers, des laboratoires publics ou privés. 


\section{A data-driven approach for predicting the remaining useful life of steam generators}

\author{
Hoang-Phuong Nguyen, William Fauriat and Enrico \\ Zio \\ Chair on System Science and the Energetic Challenge \\ CentraleSupélec, Université Paris-Saclay \\ 91192 Gif-sur-Yvette, France \\ Email: hoang-phuong.nguyen@,centralesupelec.fr, \\ william.fauriat@centralesupelec.fr, \\ enrico.zio@centralesupelec.fr
}

\author{
Jie Liu \\ School of Reliability and System Engineering \\ Beihang University \\ 37 Xueyuan Road, Haidian, Beijing, China \\ Email: liujie805@buaa.edu.cn
}

\begin{abstract}
The reliability of steam generators in nuclear power plants has always been a challenging issue. Various diagnostic models have been proposed in the literature. However, no work has been reported on the development of a robust prediction model for forecasting the future health state of steam generators. In this paper, we propose an ARIMA-based prognostic approach for tracking the degradation evolution in a steam generator and further predicting its Remaining Useful Life (RUL) before breakdown. A case study concerning real degradation datasets from different steam generators is extensively investigated to validate the performance of the proposed model.
\end{abstract}

Keywords-nuclear power plant; steam generators; prognostics and health management; ARIMA; remaining useful life.

\section{INTRODUCTION}

The need of delivering safe and economic nuclear power and extending the life of existing plants maintaining the required safety margins has led to great emphasis on the development of predictive maintenance for nuclear power plants (NPPs). This is being particularly important in France where more than $85 \%$ of electricity is currently produced from nuclear reactors, the reliability and safety of NPPs have become important issues to ensure a stable electrical supply to the whole country [1]. Among the most critical components in a NPP, steam generators (SGs), which play a key role in transferring heat from the primary side to pressurized steam on the secondary side of the plant, often suffer various degradation mechanisms, such as flow accelerated corrosion [2]-[4], stress corrosion cracking [5][8], and tube support plate (TSP) clogging or blockage [9][13]. These unanticipated failures can lead to forced plant shutdowns, with significant economic losses. For example, between 2004 and 2006 three emergency shutdowns in French NPPs due to the degradation of SGs were reported [14]. These accidents were eventually identified to be caused by clogging of the flow holes of upper TSPs. To reduce the occurrences of such unexpected catastrophic breakdowns, the development of models for detecting incipient failures and predicting the future health state of equipment has become crucial.

In the last decades, a great number of studies have been investigated to address the above problems. In [1], several simulations regarding the corrosion product deposits were carried out based on the physicochemical phenomena of TSP clogging. The results indicated that TSP clogging is a complicated process, highly influenced by a specific geometry and a large number of thermohydraulic and chemical parameters. To address this issue, Prusek et al. [9] proposed a specific model for TSP clogging including the TSP specific geometry and the induced thermohydraulic changes, which aimed to characterize and predict the localization and the growth rate of deposits during the clogging process. A fault diagnostic approach based on statistical techniques was, then, presented to quantify the clogging rate of a SG, by using the observations of its wide range level (WRL) response during a power transient [10]. Inspired by this work, Girard et al. [14] introduced a clogging rate indicator which can be used to quantify and assess the global effect of the clogging degradation on the dynamic behavior of SGs. However, most works reported in literature are concentrated on only detecting and characterizing the degradation in SGs. To the author's knowledge, no work has been done on the prediction of the future degradation evolution of SGs and their remaining useful life (RUL) before breakdown. In brief, RUL is defined as the time left before the degradation of an equipment exceeds a failure threshold [15]. An accurate RUL prediction would allow utilities to timely schedule optimal maintenance operations at a convenient time rather than to suffer a forced outage with significant losses. Moreover, the earlier the RUL is predicted, the higher the chances are with limited damage to adjacent components, increasing plant safety, and minimizing downtime and maintenance costs [16].

In this paper, we present a data-driven prognostic approach for forecasting the future trajectory of the TSP clogging rate in SGs, and further estimate the RUL before breakdown. An autoregressive integrated moving average 
(ARIMA) model is employed to model and forecast the degradation tendency by exploiting the condition monitoring data measured during the operation of SGs. Various steam generator degradation datasets from NPPs are used to validate the effectiveness of the proposed approach.

The rest of the paper is organized as follows. Section II provides a brief introduction to the steam generator considered in this study. Section III presents the proposed prognostic approach for predicting the degradation trend and the RUL of the SG. The experimental results are shown in Section IV, and Section V concludes the paper.

\section{SteAm Generator Degradation}

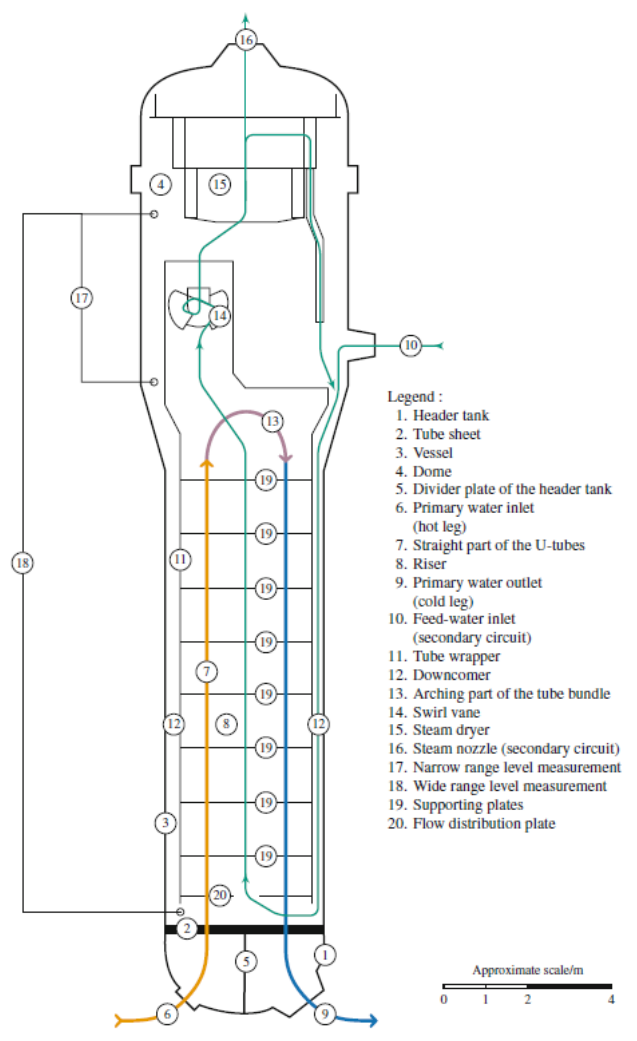

Figure 1. Schematic of a model 51B steam generator in frontal cut [14].

\section{A. Steam Generators}

Steam generators (SGs) are critical components for pressurized water reactors (PWRs), which serve as heat exchangers for feeding steam to electricity generators. In particular, each SG consists of several reserved-U tubes, between 3000 and 6000 tubes, as illustrated in Fig. 1 [14]. The primary flow comes from the nuclear reactor core through the hot leg, circulates inside the tubes and goes back to the core by the cold leg. A pressure typically equal to approximately 15.5 Mpa is maintained in this flow to avoid extended boiling phenomena in the core. Outside the tubes, the secondary flow circulates through the bundle and is transformed into steam, which flows to drive the turbines. The tubes of the SGs are supported by several plates, called tube support plates (TSPs), and flow holes allow the secondary flow to circulate through these plates. At the top of the tube bundle, the steam flow is separated into liquid phase and steam phase.

\section{B. TSP Clogging Degradation}

Particles and dissolved species produced by metal oxidation of plant components circulates, with the feedwater. Moreover, impurities and contaminants from make-up water, the auxiliary feedwater and condenser leak also contribute to the circulation in the steam flow. These circulated particles are called corrosion products and a small part of them is eliminated by the purges located at the bottom of SGs. However, most of the material which cannot be removed by the flow still remains and produces deposits, which lead to performance downgrade [17].

In short, TSP clogging is a deposit at the inlet of TSP flow holes, as illustrated in Fig. 2 [9]. This deposit phenomenon can induce high transverse velocity in the secondary flow, degrading the recirculation ratio of SGs, and eventually leading to vibrations and tubes cracks.

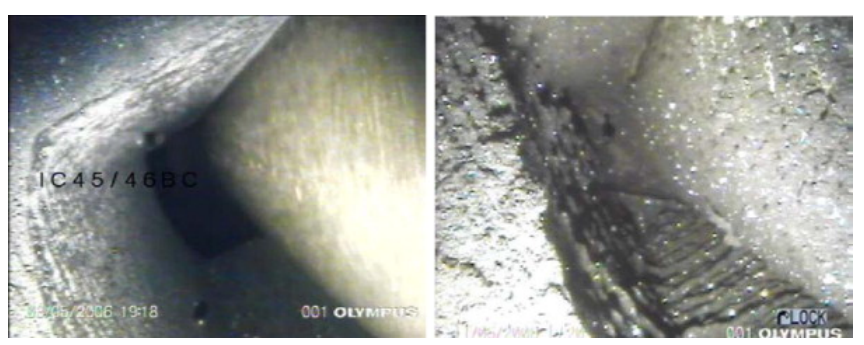

Figure 2. Tube support plate clogging: top view of a clean flow hole on the left side and an entirely blocked flow hole on the right side [9].

\section{Proposed ARIMA-BASEd Prognostic ApProACH}

\section{A. ARIMA Model}

The degradation state of SGs over time can be treated as a time series by autoregressive models. In this study, an autoregressive integrated moving average (ARIMA) model [18] is employed to identify the underlying structure in the acquired data and further forecast the future states of degradation. 


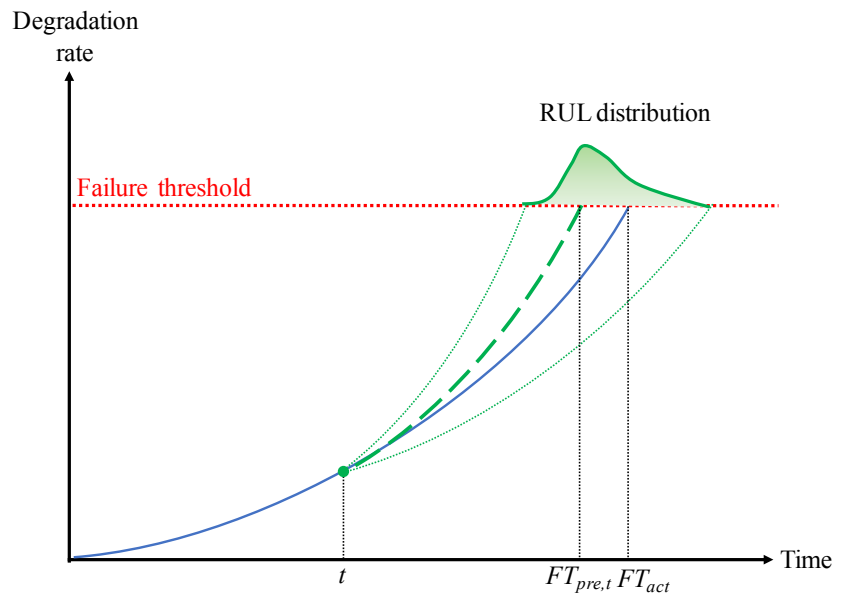

(a)

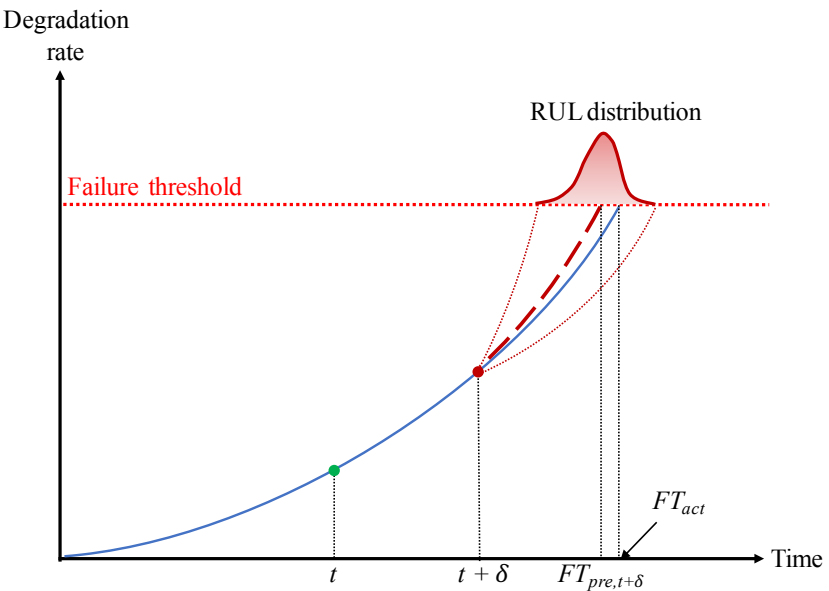

(b)

Figure 3. An illustration of the RUL estimation scheme: (a) Prediction at time t; (b) Prediction at time $\mathrm{t}+\delta$.

In general, an ARIMA model is specified by a combination of three order parameters: $(p, d, q)$, which correspond to the following processes:

1) Autoregressive (AR) component: refers to the use of past values in the regression model for the actual series $X$. The autoregressive parameter $p$ specifies the number of lags used in the model.

2) Integrated (I) component: represents the degree of differencing, specified by the integrated parameter $d$. The integrated component can also be used to stabilize the series when the stationary assumption is not met.

3) Moving average (MA) component: indicates the error of the model as a combination of previous errors. The order $q$ specifies the number of lagged forecast errors used in the model.

Autoregressive, differencing, and moving average components make up a non-seasonal ARIMA model which can be defined as follows:

$$
\phi(L) \cdot(1-L)^{d} \cdot x(t)=\theta(L) \cdot \varepsilon(t)
$$

where

$$
\left\{\begin{array}{c}
\phi(L)=1-\phi_{1} L-\phi_{2} L^{2}-\ldots-\phi_{p} L^{p} \\
\theta(L)=1-\theta_{1} L-\theta_{2} L^{2}-\ldots-\theta_{q} L^{q} \\
x(t) \cdot L=x(t-1)
\end{array}\right.
$$

where $x(t)$ is the actual degradation state series, $\left\{\phi_{p}\right\}$ and $\left\{\theta_{q}\right\}$ are the parameters of the AR and MA components, respectively, $L$ is the lag operator, $\varepsilon(t)$ is the random error at time $t$.

To develop an ARIMA model, the three following steps are repeated until a satisfactory model is obtained:
1) Model identification: values for the orders of the autoregressive, differencing, and moving average components, namely $p, d$, and $q$, respectively, are hypothesized using a training set of time series data. A fitness criterion is also defined.

2) Parameter estimation: using non-linear optimization techniques (e.g. least-squares method), hyperparameters of the ARIMA equations are estimated for minimizing the overall error between model output and measured data. In order to efficiently identify the optimal hyperparameters of the ARIMA model, a random search optimization strategy [19] is used in this study.

3) Model validation: several standard diagnostic metrics (e.g. Akaike Information criterion (AIC), Bayesian Information criterion (BIC), and final prediction error (FPE)) can be used to evaluate the adequacy of the ARIMA model. In this work, AIC is used for evaluating the goodness-of-fit of the model.

\section{B. Degradation Trajectory Prediction and RUL Estimation}

The process of predicting future degradation states and estimating the RUL is illustrated in Fig. 3. The blue solid line represents actual observations of degradation measured in time.

At time $t$, the historical observations $\left\{x_{i}, i=1, \ldots, t\right\}$ are employed for optimizing the hyperparameters of the ARIMA model. The optimized model is, then, used to generate a forecasted degradation trajectory until reaching the prediction horizon $T$, as depicted by the green dashed line in Fig. 3(a). The RUL at time $t, R U L_{\text {pre,t }}$, can be defined as the time required for the forecasted trajectory to reach the failure threshold, as follows:

$$
R U L_{p r e, t}=F T_{p r e, t}-t \text {, }
$$


where $F T_{\text {pre,t }}$ is the failure time forecasted at the prediction time $t$. Note that the future degradation states and the RUL are predicted using only the past observations, because future observations $\left\{x_{t+k}, k=1, \ldots, T-t\right\}$ have not been collected yet.

At the next prediction period $(t+\delta)$, where $\delta$ is the time interval between two consecutive periods ( $\delta=3$ months in the following case study), the hyperparameters of the ARIMA model are updated when the new measurements are available. Using this updated model, a new degradation trajectory (the red dashed line in Fig. 3(b)) and the RUL at time $(t+\delta), R U L_{p r e, t+\delta}$, are predicted. This update process is continued in this manner until the end of the time horizon $T$. Finally, a multistep-ahead prediction of the degradation trajectory is obtained by concatenating the forecasted trajectories at each prediction time across the entire life of the SG.

\section{RESULTS \& DisCUSSION}

In this section, a case study is carried out to validate the developed prognostic approach, including the degradation data of three different SGs. These datasets were acquired as the result of a TSP clogging diagnosis. The degradation of the SGs were obtained over a period of 15 years, with a total number of 5000 observations, as shown in Fig. 4, which corresponds to the operating period of the considered SGs before the chemical cleaning performed in 2006 [14]. In Fig. 4 , the vertical axis represents the TSP clogging percentage rate of the SGs, ranging from $0 \%$ to $100 \%$. Based on the expert judgement, a failure threshold is defined at $80 \%$ of the degradation rate, which corresponds to the critical clogging level with a substantial risk of tube cracking [14].

To evaluate the performance of the proposed approach, three popular forecast accuracy metrics are considered: a) Root Mean Square Error (RMSE); b) Mean Absolute Percentage Error (MAPE); c) Mean Absolute Scaled Error (MASE) [20]. The definitions of these metrics are, respectively, given as follows:

$$
\begin{gathered}
R M S E=\sqrt{\frac{1}{N} \sum_{i=1}^{N}\left(\hat{x}_{i}-x_{i}\right)^{2}} \\
M A P E=\frac{1}{N} \sum_{i=1}^{N}\left|\frac{\hat{x}_{i}-x_{i}}{x_{i}}\right|
\end{gathered}
$$

$$
M A S E=\frac{1}{N} \sum_{i=1}^{N}\left(\frac{\left|\bar{x}_{i}-x_{i}\right|}{\frac{1}{N-1} \sum_{j=2}^{N}\left|x_{j}-x_{j-1}\right|}\right),
$$

where $N$ is the number of observations, $x$ and $\hat{x}$ are the actual and predicted degradation states, respectively.

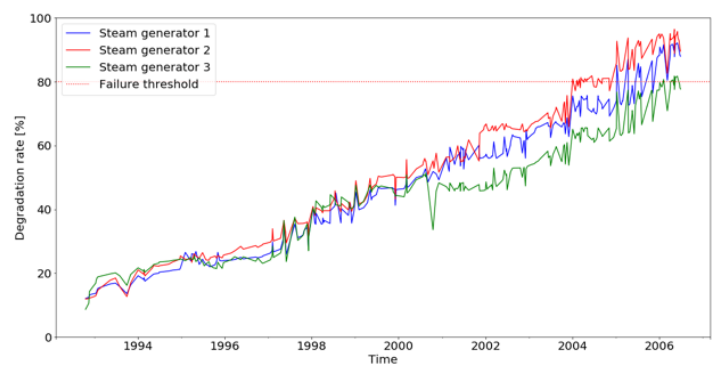

Figure 4. Raw degradation series of steam generator Nos. 1-3.

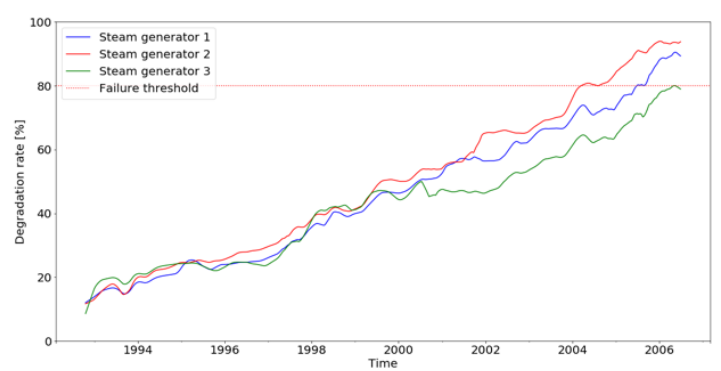

Figure 5. Pre-processed degradation series of steam generator Nos. 1-3 by LOWESS.

To reduce the unwanted noise in the measured data, a local regression filter, called Locally Weighted Scatterplot Smoothing (LOWESS) [21], is adopted to preprocess the datasets with a span value of $1.5 \%$. The preprocessed results are shown in Fig. 5.

To perform the proposed prognostic approach, each dataset is initially divided into two different subsets: a training dataset and a test dataset. In this study, the first prediction starting point is set to a specific date of January $1^{\text {st }}, 2002$. The observations before the prediction starting point, for each SG, are used as the training dataset for developing the prediction model, while the remaining data are reserved as the test dataset, which is employed for evaluating the effectiveness of the proposed approach. At each prediction period, new collected measurements are used to timely update the hyperparameters of the prediction model and the forecasted degradation trajectory as described in Section III. The 
forecasted RUL of the equipment is also updated at each prediction period. An illustration of the degradation trajectory prediction of the proposed model at the $3^{\text {rd }}$ and $10^{\text {th }}$ prediction periods for SG No. 1 is shown in Fig. 6.
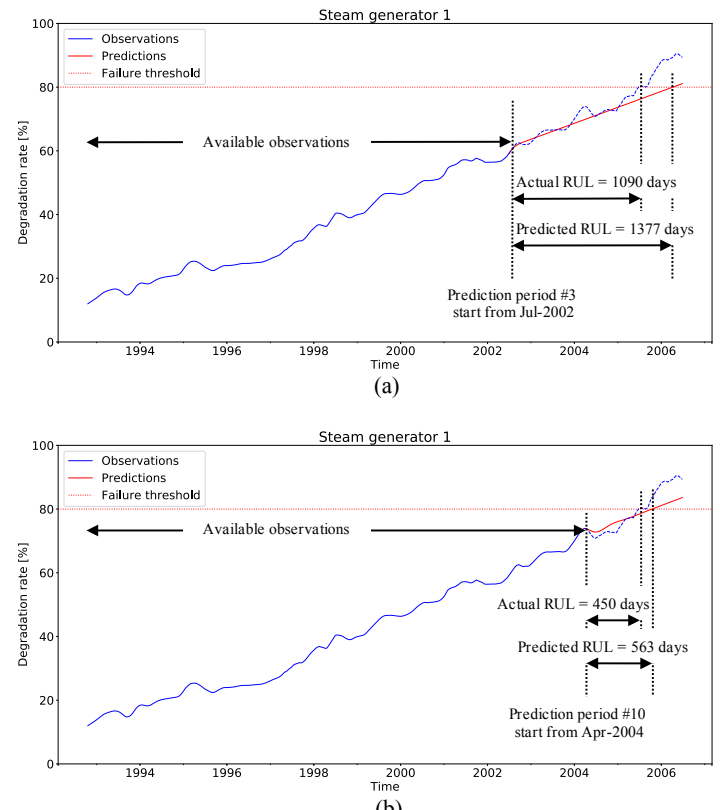

Figure 6. Degradation trajectory prediction for steam generator No. 1: (a) At the $3^{\text {rd }}$ prediction period; (b) At the $10^{\text {th }}$ prediction period.

Figs. 7 and 8 show the multistep-ahead prediction and the RUL estimation of the proposed approach across the lifetime of SG No. 1. As shown in these Figures, the proposed approach exhibits satisfactory performance in accurately predicting the degradation trajectory and the RUL of the considered SG. This is due to the advantageous capability of the proposed prediction model in dynamically updating knowledge about the current degradation trend of the target SG when new measurements come.

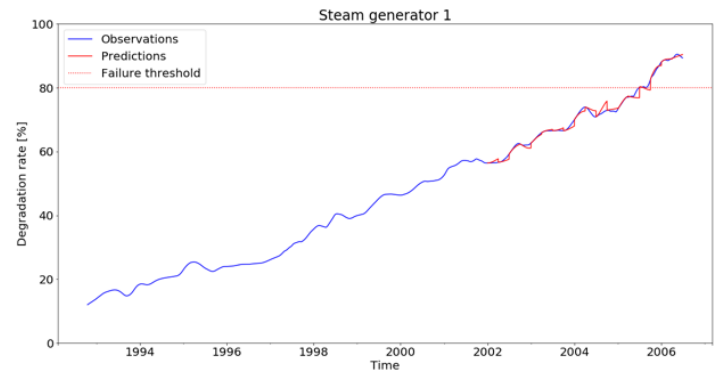

Figure 7. Degradation trajectory prediction for steam generator No. 1.

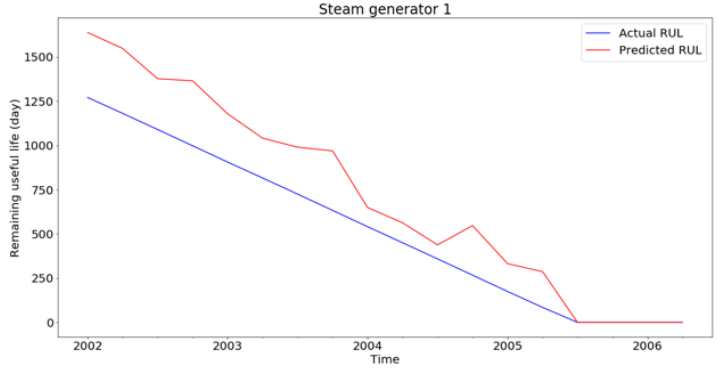

Figure 8. RUL prediction for steam generator No. 1.

Figs. 9 and 10 show the results of exploiting the proposed model for other SGs, and performance comparisons in terms of prediction accuracy is given in Tables 1 and 2. In Table 2, the error metric represents the percentage error between the actual RUL and the predicted RUL at the same date, defined as follows:

$$
\text { Error }_{t}=\frac{R U L_{a c t}-R U L_{p r e, t}}{R U L_{a c t}} \times 100 \%
$$

where $R U L_{a c t}$ is the actual RUL of the SGs. The results clearly show that the proposed approach has achieved an excellent performance throughout the entire lives of different SGs. As can be seen in Table 2, the prediction performance of the proposed model, for all considered SGs, becomes more accurate when more available measurements come. In particular, at the prediction point in January 2006, the proposed model can completely predict the true RUL of the SG No. 3 without error.

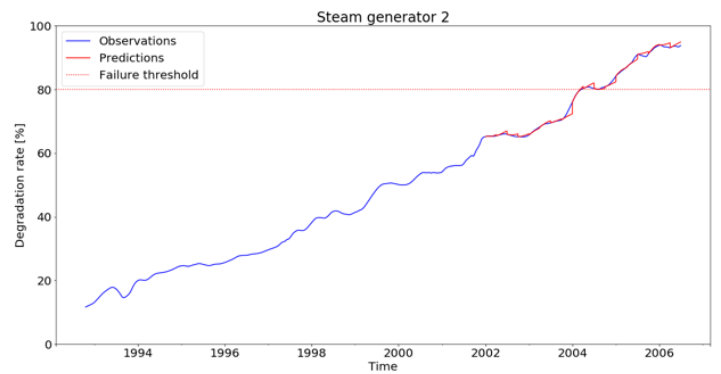

Figure 9. Degradation trajectory prediction for steam generator No. 2 . 


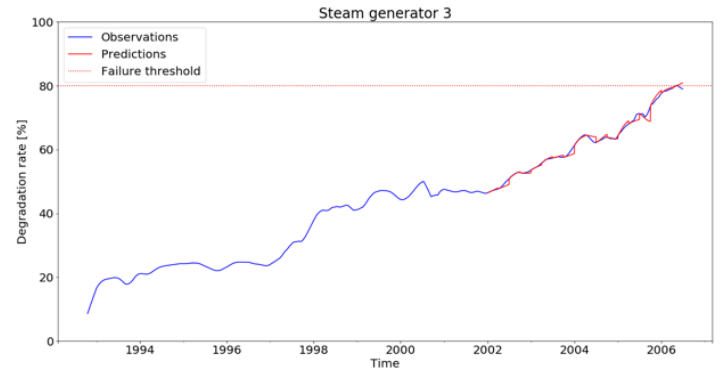

Figure 10. Degradation trajectory prediction for steam generator No. 3.

TABLE I. Performance Evaluation Of Degradation Evolution PREDICTION.

\begin{tabular}{cccc}
\hline Testing dataset & RMSE & MAPE & MASE \\
\hline SG_1 & 0.83 & 0.74 & 19.13 \\
SG_2 & 0.65 & 0.50 & 18.41 \\
SG_3 & 0.81 & 0.75 & 18.35 \\
Average & 0.76 & 0.67 & 18.63 \\
\hline
\end{tabular}

TABLE 2. Performance EVAluation of RUL PREdiction.

\begin{tabular}{ccccc}
\hline $\begin{array}{c}\text { Testing } \\
\text { dataset }\end{array}$ & Current date & $\begin{array}{c}\text { Actual RUL } \\
\text { (day) }\end{array}$ & $\begin{array}{c}\text { Predicted } \\
\text { RUL (day) }\end{array}$ & Error (\%) \\
\hline \multirow{2}{*}{ SG_1 } & Jan-2002 & 1271 & 1638 & 28.87 \\
& Jul-2004 & 359 & 438 & 22.00 \\
\hline \multirow{2}{*}{ SG_2 } & Jan-2002 & 803 & 1007 & 25.40 \\
& Jan-2004 & 73 & 65 & 10.96 \\
\hline \multirow{2}{*}{ SG_3 } & Jan-2002 & 1580 & 1638 & 3.67 \\
& Jan-2006 & 119 & 119 & 0 \\
\hline
\end{tabular}

\section{CONCLUSIONS}

In this paper, a prognostic model based on ARIMA is proposed to predict the evolution of the TSP clogging degradation in steam generators. The advantage of the proposed model lies in the capability to adaptively and timely update its prediction performance when new measurements are collected. The results indicate that the proposed prognostic model is able to accurately predict the future trajectory of the TSP clogging rate and the RUL across different steam generators.

\section{REFERENCES}

[1] G. Yang, V. Pointeau, E. Tevissen, and A. Chagnes, "A review on clogging of recirculating steam generators in Pressurized-Water
Reactors," Prog. Nucl. Energy, vol. 97, pp. 182-196, 2017.

[2] B. Chexal, J. Horowitz, and B. Dooley, "Flow-accelerated corrosion in power plants. Revision 1." 1998.

[3] V. Kain, S. Roychowdhury, T. Mathew, and A. Bhandakkar, "Flow accelerated corrosion and its control measures for the secondary circuit pipelines in Indian nuclear power plants," J. Nucl. Mater., 2008.

[4] X. X. Yuan, M. D. Pandey, and G. A. Bickel, "A probabilistic model of wall thinning in CANDU feeders due to flow-accelerated corrosion," Nucl. Eng. Des., 2008.

[5] R. W. Staehle and J. a. Gorman, "Quantitative Assessment of Submodes of Stress Corrosion Cracking on the Secondary Side Steam Generator Tubing in Pressurised Water Reactors: Part 1," Corrosion, 2003.

[6] V. N. Shah et al., "Assessment of primary water stress corrosion cracking of PWR steam generator tubes," Nucl. Eng. Des., 1992.

[7] P. M. Scott, "An overview of materials degradation by stress corrosion in PWRs," in Corrosion Issues in Light Water Reactors: Stress Corrosion Cracking, 2007.

[8] U. C. Kim, K. M. Kim, and E. H. Lee, "Effects of chemical compounds on the stress corrosion cracking of steam generator tubing materials in a caustic solution," in Journal of Nuclear Materials, 2005

[9] T. Prusek, E. Moleiro, F. Oukacine, A. Adobes, M. Jaeger, and M. Grandotto, "Deposit models for tube support plate flow blockage in Steam Generators," Nucl. Eng. Des., vol. 262, pp. 418-428, Sep. 2013.

[10] S. Girard, T. Romary, F. Jean-Melaine, P. Stabat, and H. Wackernagel, "Sensitivity analysis and dimension reduction of a steam generator model for clogging diagnosis," Reliab. Eng. Syst. Saf., vol. 113, pp. 143-153, May 2013.

[11] M. Guillodo et al., "Experimental and numerical study of deposit formation in secondary side SG TSP by electrokinetic approach." 01-Sep-2012.

[12] P. Bertrand, N. Gay, and R. [Electricite D. F. (France)] Crinon, "EDF steam generators fleet: In-operation monitoring of TSP blockage and tube fouling," 2012.

[13] A. Vergnault, R. Dorel, and F. Goux, "Steam Generator Blockage: A Thermal-Hydraulic Approach Based on CATHARE 2," no. 48167. pp. 381-387, 2008.

[14] S. Girard, "State of the Art of Clogging Diagnosis BT - Physical and Statistical Models for Steam Generator Clogging Diagnosis," S. Girard, Ed. Cham: Springer International Publishing, 2014, pp. 1524.

[15] X. S. Si, W. Wang, C. H. Hu, and D. H. Zhou, "Remaining useful life estimation - A review on the statistical data driven approaches," Eur. J. Oper. Res., 2011.

[16] K. Javed, R. Gouriveau, N. Zerhouni, and P. Nectoux, "Enabling health monitoring approach based on vibration data for accurate prognostics," IEEE Trans. Ind. Electron., 2015.

[17] G. Corredera, M. Alves-Vieira, and O. de Bouvier, "Fouling and TSP Blockage of Steam Generators on EDF Fleet: Identified Correlations with Secondary Water Chemistry and planned Remedies," in International Conference, Water chemistry of nuclear reactor systems; 2008; Berlin, Germany, 2008.

[18] G. E. P. Box and G. M. Jenkins, Time Series Analysis: Forecasting and Control. 1994.

[19] J. Bergstra JAMESBERGSTRA and U. Yoshua Bengio YOSHUABENGIO, "Random Search for Hyper-Parameter Optimization," J. Mach. Learn. Res., 2012.

[20] R. J. Hyndman and A. B. Koehler, "Another look at measures of forecast accuracy," Int. J. Forecast., 2006.

[21] J. Iwaniec, W. Lisowski, and T. Uhl, "Nonparametric approach to improvement of quality of modal parameters estimation," Jour. Theor. Appl. Mech., vol. 43, pp. 327-344, 2005. 